\title{
THE RELATIONSHIP BETWEEN DRIVER DISTRACTION AND MENTAL WORKLOAD
}

\author{
T.W. Schaap ${ }^{1 *}$, A.R.A van der Horst $^{2}$, B. van Arem $^{3}$, K.A. Brookhuis ${ }^{4}$ \\ 1*. University of Twente, Centre for Transport Studies, Research Centre AIDA; \\ P.O. Box 217, 7500 AE Enschede, The Netherlands; +31534894361; T.W.Schaap@UTwente.nl \\ 2. TNO Defence, Security and Safety, BU Human Factors, Soesterberg, The Netherlands \\ 3. Delft University of Technology, Civil Engineering and Geosciences, Transport \& Planning, Delft, \\ The Netherlands; \\ 4. Delft University of Technology, Faculty of Technology, Policy \& Management, Delft, The \\ Netherlands; University of Groningen, Faculty of Behavioural and Social Sciences, Groningen, The \\ Netherlands
}

\begin{abstract}
Driver distraction is caused by a competing activity and leads to unsafe driving. Mental workload changes with task demands and influences performance. Though distraction and mental workload are strongly related, they are not the same. Performance motivation and task engagement influence performance and consequently distraction but not workload; environment complexity and driver state influence mental workload but not distraction. Although distraction can be manifested in directly observable unsafe driving, readiness to respond and event detection are also important aspects of driving performance. The Peripheral Detection Task, used for workload assessment, can be used for assessing this latent form of distraction.
\end{abstract}

\section{KEYWORDS}

Driver distraction, Mental workload, Peripheral Detection Task, Event detection, Readiness to respond, Engagement 


\section{INTRODUCTION}

Driver distraction is a research topic that has been in the spotlight for many years, because of its impact on traffic safety conflicts. The American National Highway Traffic Safety Administration (NHTSA) has estimated that in approximately $25-30 \%$ of traffic accidents, driver distraction is a contributing factor [1]. Driver distraction manifests itself in many ways, such as eating and drinking, tuning the radio, engaging in a conversation with a passenger or through a mobile phone, entering a destination in a navigation system or dialling a telephone number [2].

The strong increase in mobile phone use while driving has served as a catalyst to driver distraction research, and with good reason. Epidemiological studies showed that the likelihood of being involved in a conflict or accident while using a mobile telephone was four times as high compared to not using a mobile telephone [3,4]. The Dutch Institute for Road Safety Research SWOV calculated that in 2004 nearly 600 victims of traffic accidents could have been saved if mobile phone use while driving were banned, i.e. approximately $8 \%$ of all registered hospitalized and fatally injured in the Netherlands [5]. Furthermore, some studies show that mobile phone users wear their seatbelts significantly less than other drivers, leading to an increase in the seriousness of injuries as a consequence of traffic accidents (e.g., [6]).

Whereas these numbers focus merely on the risks of mobile phone use, driver distraction in the broader sense presents a general risk for traffic safety. The driving task is complex and demanding in terms of visual and cognitive attention. Without focused attention on the primary task, drivers are at risk of responding more slowly or less appropriately to complex or changing situations that require their full attention (e.g., [7, 8, 9, 10]). Furthermore, drivers have difficulty assessing their own driving performance, especially while performing highly engaging distracting tasks [11]. Finally, even though many distracting tasks within the vehicle, such as dialling or eating, are initiated by drivers themselves, they do not strategically postpone these distractions [12]. This all provides good reason for studying the effects of and mitigation strategies for distraction while driving.

The challenge of studying distraction is that it is not easily measured. A certain degree of distraction may lead to a decrease in driver performance. However, this decrease does not always materialize, as the driver may be able to cope with doing multiple tasks simultaneously in the current driving situation. This paper aims to overcome this limitation by using a related concept: mental workload. We will explore the similarities and differences of both terms and analyze to what extent studies on driver distraction can benefit from the assessment methods that are used in mental workload research.

\section{PREVIOUS STUDIES INTO DRIVER DISTRACTION}

Many studies into the causes of driver distraction, its effects on driver performance, and strategies for reduction or mitigation of these effects have already been conducted (for an overview of recent studies, see [13]). In most of these studies, participants were asked to perform a secondary task while driving, to study the effects of distraction on driver performance. The driving task may, for instance, be combined with simultaneous use of a mobile phone, either hands-free or handheld, for a simulated conversation. For example, in one driving simulator study in which participants drove a 30 kilometre section of motorway in normal traffic, a lead car suddenly decelerated [7]. Participants drove in three conditions, i.e. 
while keeping their visual attention focused on the car in front (baseline condition), while performing a visually loading task using a numeric keypad, or while performing a cognitively loading task representing engaging in a conversation over a mobile phone. Driver performance, as measured by time-to-collision and brake reaction time, was impaired in both the visual-distraction condition and the cognitive distraction condition [7]. In another study into the effects of mobile phone use [14], the effects of carrying out secondary tasks of varying cognitive complexity on drivers' visual behaviour and subjective perception of safety and mental workload were studied. The secondary tasks were communicated to the participants through a hands-free mobile telephone. Participants drove an instrumented car in a baseline condition (no secondary task), in a low cognitive distraction condition (while completing easy addition problems), and in a high cognitive distraction condition (while solving more complex arithmetic problems). This study reveals that drivers made significantly less high-speed saccadic eye movements and spent less time looking to the right periphery for impending hazards with increasing complexity of the secondary tasks. Drivers also spent less time checking their mirrors and instruments. Finally, with the difficulty of the secondary tasks, drivers' subjective rating of workload and distraction increased [14].

In another study, the effects of two surrogate in-vehicle information systems (S-IVIS) on driver behaviour in an urban environment were investigated [10]. The interaction with other vehicles was studied when using a visual S-IVIS (visually distracting) and an auditory S-IVIS (cognitively distracting). Results showed that drivers interacted with other vehicles less appropriately and that the interaction with vulnerable road users also suffered due to increased intersection approach speed. This speed increase was more frequent with the cognitively distracting S-IVIS than with the visually distracting task [10].

Finally, a recent simulator experiment [15] was conducted to determine the effects of iPod ${ }^{\mathrm{TM}}$ use and practice on driving performance during safety-critical situations. It was found that perception-response times and number of collisions increased during performance of the difficult secondary task, which was searching for a particular song in the iPod ${ }^{\mathrm{TM}}$ menu. Safe driving performance increased with practice, although it remained negatively affected relative to the baseline condition [15].

\section{DRIVER DISTRACTION: THEORY AND DEFINITION}

When studying a complex concept such as driver distraction, stemming from many sources and manifesting itself in many forms, uniformity in definitions is an important constraint for clear and unambiguous research results. Driver distraction is a subset of driver inattention, a situation in which the primary driving task is performed without complete, focused attention on that driving task. Whereas inattention can also occur without a distracter just by no longer paying attention, distraction is related to something (a task, object or person) that draws away the attention that is needed to perform the driving task adequately.

Unfortunately, the research community has not until this point shown consensus on the precise definition of driver distraction. The definitions used so far vary from "Diversion of attention from the driving task that is compelled by an activity or event inside the vehicle" [16], which is completely confined to the cause of the distraction inside the vehicle, to " $A$ disturbance imposed within a lateral or longitudinal control vehicle loop" [17], focusing on the possible effects on vehicle control. A rather extensive overview of definitions of driver distraction recently used in human factors research is given in [18]. This overview shows the 
large variation between definitions, and how long the research community has already been searching for a uniform definition of the construct. This variation in theoretical definitions leads to difficulties in the uniform operationalization of the construct of distraction. Is distraction determined by the sole presence of a task, object or event that does not directly contribute to adequate driving, by the decrease in attention to the driving task, or by reduced performance on the primary driving task? The answer to such questions is needed to operationalize the concept of distraction, which in turn is the key to comparing the different studies and their results on this subject.

Defining distraction in a broad way creates a complete definition that is difficult to operationalize, whereas a definition which incorporates strictly defined behavioural effects is on the one hand very clear for all, but may on the other hand fail to capture important aspects of distraction [18]. The definition that is proposed in [18], based on an overview of definitions is the following:

"Driver distraction is a diversion of attention away from activities critical for safe driving toward a competing activity”.

This definition makes clear that safe driving performance deteriorates when the driver is distracted. Attention that is crucial for driving safely is diverted away by a distracting task, leaving too few resources available for adequate performance in the primary driving task.

The measurable effect of driver distraction is a decrease in driving performance. The most commonly used measures for driver distraction are longitudinal control (speed and headway measures), lateral control (time-to-line crossing and SD lateral position), event detection and gap acceptance performance [19]. These measures may indicate that the driving performance deteriorates. Particularly if a negative effect is seen on more than one of the above measures during the performance of a secondary task, it can be concluded that driving performance deteriorates due to distraction.

However, safe driving can not always be determined solely on the basis of directly observable measures. Driving safely also requires that the driver is ready to respond adequately to possible upcoming safety-critical situations, and distraction can affect this readiness. If this is the case, and a driver is in such a (distracted) state that his readiness to respond to an upcoming hazard is negatively affected, this form of decreased performance does not show in measures of operational driving behaviour when the complexity of the driving environment is low. However, it can be argued that the driver is no longer driving optimally. Looked at it in this way, the presence of a secondary task can lead to three possible situations, each with a different effect on driving performance. Below, these situations will be briefly described and it will be determined whether and how distraction can be measured.

i. No distraction

The presence of a secondary task does not distract the driver from driving safely per se. The driver can choose to give full priority to the primary driving task without letting the secondary task affect the level of driver performance. Although this may lead to poor performance on the secondary task, distraction from the primary driving task does not occur. Even in safetycritical situations, the driver will be able to respond appropriately and in a safe manner.

ii. Manifest distraction 
In the second situation the presence of a secondary task leads to such distraction that a measurable decrease in driver performance can be observed directly. The driver makes suboptimal decisions and the number of conflicts increases. In this situation, distraction can be assessed directly through measuring its impact on driving behaviour in terms of operational driving tasks and conflict measures. By looking at conflict situations, defined as "observable situations in which two or more road-users approach each other in time and space to such an extent that there is a risk of collision if their movements remain unchanged" [20], a statement can be made about the likelihood of an accident. The seriousness of a conflict can be determined by surrogate safety measures $[21,22]$. Two key surrogate safety measures are the Time-To-Collision (TTC), and Post-Encroachment-Time (PET) [21]. PET is defined as the time that elapses between the moment that the first vehicle leaves the (future) path of the second vehicle and the moment that the second vehicle reaches the path of the first vehicle no collision course is necessary [21].

\section{iii. Latent distraction}

In the third situation, directly observable measures of driving performance such as operational driving measures or conflict measures seem to indicate safe driving in the presence of a secondary task. However, this does not definitively establish that the driver is not distracted. After all, driving safely does not simply mean that the driver is not in an unsafe situation, but also that he can adequately respond to upcoming safety-critical situations in his current state. In a situation with latent distraction, a driver can manage the current situation, but at the limit of his/ her capacity, and would therefore struggle to respond to a suddenly upcoming safetycritical situation. While distraction can therefore indeed be present, the current assessment methods are unable to establish or measure it. It would be useful to identify a way of predicting whether a driver is affected by this form of distraction. In this paper we aim to find alternative assessment methods for this situation.

A concept strongly related to driver distraction is mental workload. Increased mental workload is also often used as a direct indication of distraction [19], but we will show that this practice is not always correct. Mental workload is not the same as driver distraction, but the concepts are similar in the way that a secondary task and the way in which task demands and task goals are dealt with play an important role. That is why certain measures of mental workload may have value in indicating distraction.

\section{MENTAL WORKLOAD}

De Waard [23] conducted extensive research into the concept of mental workload and the measures that can be used for its assessment. He distinguishes between task demands, task complexity, task difficulty, and mental workload. Task demands are determined by the end goal of the task. Task complexity is related to a number of stages that have to be completed in order to successfully attain the end goal. Finally, task difficulty deals with the ability of the operator to execute the task, depending on the state of the operator. While in normal circumstances it might be easy for a driver to cover a certain stretch of road, it may be more difficult to accomplish the same level of performance on the same route after a late night party.

He defines mental workload as follows: 
"Mental workload is the specification of the amount of information processing capacity that is used for task performance" [23]

De Waard shows that mental workload depends on a number of circumstances and can vary between individuals and over time, and he describes the effects of task demands on mental workload [23].

Figure 1 depicts the model of the overall relationship between workload, task demand, and performance, as presented in [23].

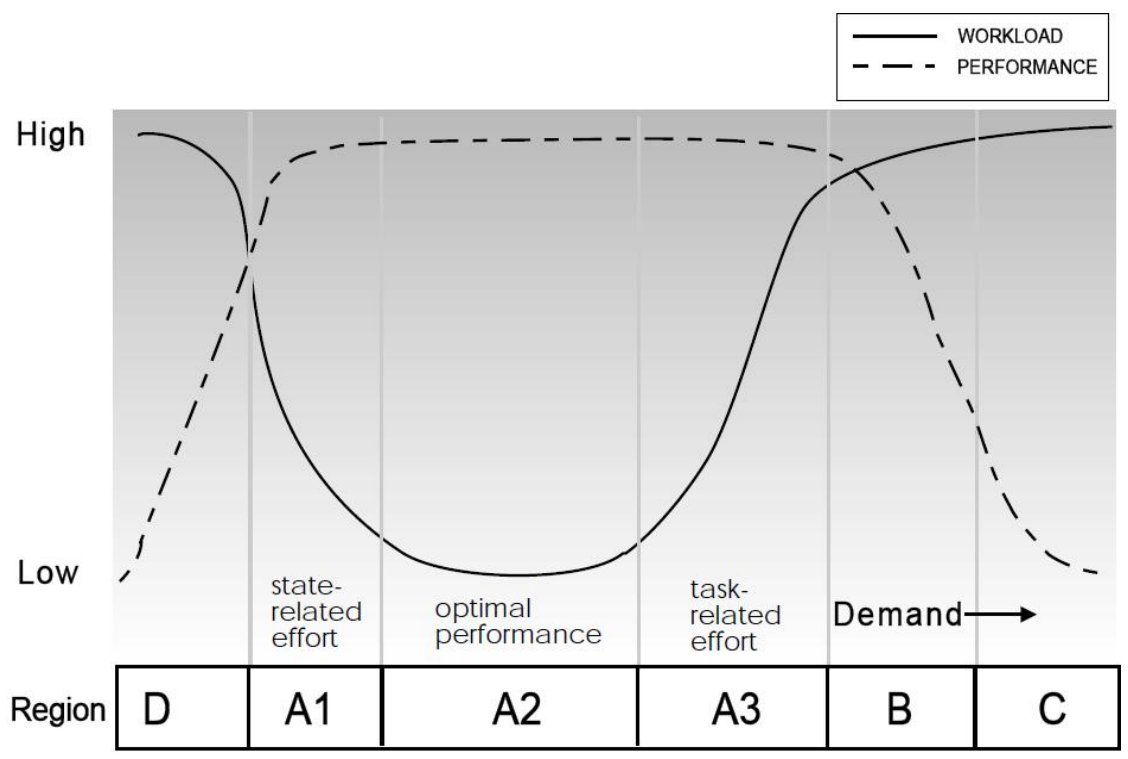

Figure 1 - Workload and performance in 6 regions (after [23])

The workload-performance relation has been separated into 6 regions, each with its own characteristics. In the three A-regions (A1, A2 and A3), the demands of the task are at a level that allows the operator to achieve a high level of performance. In the A2-region, demand and workload are optimal, and performance can easily reach the desired level. In A1, performance remains adequate, but the operator has to exert state-related effort (i.e., computational effort through controlled information processing) to keep performance high; in A3, the invested effort invested is task-related (i.e., compensatory effort). In the regions B, C and D, the operator is affected by the level of demand, leading to suboptimal performance. In region $\mathrm{B}$, demand and workload are at a level that task-related effort no longer increases performance, and performance declines. Region $\mathrm{C}$ depicts overload of the operator, leading to a low performance level. In region $\mathrm{D}$ ( $\mathrm{D}$ for de-activation), the lack of task demand coincides with a high workload level that the operator cannot cope with, so that performance deteriorates [23].

Since mental workload and distraction are both related to the reaction to the secondary task in terms of performance, it is useful to explore the options of using a number of mental workload assessment methods for distraction research.

There are a number of ways in which mental workload can be measured, and some of these measures might be useful for an indication of distraction effects. The effects of increased mental workload can differ from one situation to another and from one individual to another. 
These differences depend largely on individual strategies and the region of performance involved, and measurements of the effects are best combined for a valid assessment of mental workload [23]. Due to either increased task demands or changes in driver state, drivers can feel a subjective increase in mental workload, can show physiological signs that stem from increased mental workload, and their task performance can decrease. Also, a decrease in performance of secondary tasks can occur. By combining the assessment results an overall indication of mental workload and driver state can be determined.

\section{Primary task performance}

Increased mental workload can impair driving performance in certain situations. The measures which are the most sensitive to increases in mental workload are the Standard Deviation of Lateral Position (SDLP), and the Standard Deviation of Steering Wheel Movements (SDSWM) [23].

Compensatory driving behaviour can also be found, and is often seen as a way to reduce the demands of the driving task. When task demands increase, a decrease in driving speed can be found [e.g., 24, 25]. However, in a study described in [26], no evidence was found that drivers adjust their safety margins during tactical driving.

\section{Secondary task performance}

Any trade-off between driving performance and secondary task performance depends highly on the instructions given to the driver and the level of obtrusiveness of the secondary task. Embedded tasks are considered to be the best secondary tasks for assessment of the mental workload stemming from a certain driving situation, due to their low primary task intrusion [23]. Two examples of such embedded tasks are car-following and mirror checking, and an increase in workload can be determined by a delay in these tasks.

There are however secondary tasks that are not obtrusive and are also not embedded in the driving task, such as the Peripheral Detection Task (PDT). The PDT is based on the fact that visual attention narrows as workload increases [27]. Originally, the PDT was applied in a driving simulator setting with the stimulus presented on the simulator screen. In later studies, drivers wear a headband with a LED in their peripheral field, so that the LED moves with the drivers' head movements [28]. The LED lights up randomly every 3 to 5 seconds. Drivers also wear a small switch attached to their index finger (Figure 2), which they are instructed to press every time they notice the LED light. In general, the index finger of the dominant hand is chosen for this, unless the experimental setup does not allow for this (e.g., with manual transmission).

The PDT has been shown to be reliable to the demands of the driving task [27] and is also sensitive to peaks in workload [29], which is important for driving safety research due to changes in task demands. 

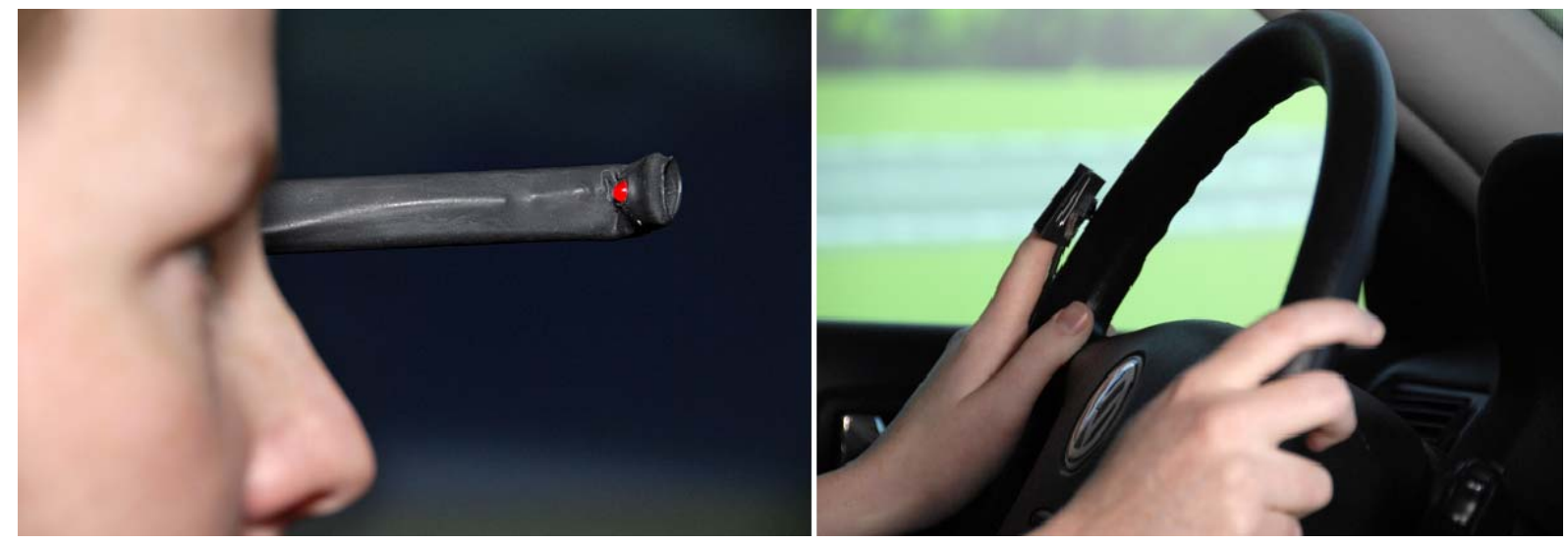

Figure 2 - Headband with LED in peripheral vision of participant (left); PDT switch on left index finger (right)

\section{Subjective measures}

Operators can experience their mental workload and give a subjective evaluation. These selfreport measures are questionnaires that were developed to measure workload in humancomputer interaction, but can be used for many interaction tasks as well, such as driving. For instance, in the NASA Task Load Index (NASA-TLX) questionnaire [30], drivers give a subjective rating of experienced workload on six rating scales: Mental Demands, Physical Demands, Temporal Demands, Own Performance, Effort, and Frustration [30]. These multidimensional ratings can be combined to calculate an overall level of perceived mental workload. Another example of a self-report measure is the RSME, the Rating Scale Mental Effort [31]. It is a single-dimensional measurement of invested mental effort.

The main disadvantage of self-report measures of mental workload is that they are no objective measurements [32], although their face validity is high [23]. Also, only an overall rating of workload over a whole drive can be given without disturbing drivers in their primary task, so a focus on specific situations is not possible. However, self-report measures give a clear indication of the level of workload perceived by drivers, and are cheap and easy to apply. They can therefore be useful to include in almost any workload-related research.

\section{Physiological measures}

When an operator experiences an increase in mental workload, the response is also reflected in physiological measures. For example, operators can have more dilated pupils, start sweating, and show increased blood pressure, heart rate and heart rate variability. An important advantage of determining the physiological effects of increased mental workload is the unobtrusiveness of most of the measurements involved [23]. However, physiological measurements require complex interpretation of results, and may also reflect emotional strain and physical activity in addition to mental workload [29]. Heart rate gives an indication of overall workload, whereas heart rate variability is more useful for determining cognitive, mental workload [33].

In order to get a reliable assessment of mental workload, it is important to combine different workload measures, since not all workload measures are sensitive to workload levels in the same regions [23]. Increased task demands and an increase in complexity of the environment 
both have an effect on the self-report scale RSME, and on the physiological measure ECG. Secondary-task performance, in particular the embedded task of car-following, is also sensitive to both sources of increased workload. For primary-task measures, this effect is not the same. While additional tasks lead to a decrease in SDLP and SDSTW, an increase in complexity of the environment increases both measures. Finally, heart rate variability is mostly sensitive to an increase in complexity [23].

\section{APPLYING MENTAL WORKLOAD MEASURES TO DRIVER DISTRACTION RESEARCH}

As mentioned above, the measures of workload can be combined into an overall rating of mental workload. But, would it be possible to use them to measure latent distraction? To do so, it must be possible to determine how well drivers are performing their task of safe driving, based on the measures for event detection and readiness to respond to an upcoming hazard. Below we will evaluate the different workload measures in terms of their ability to assess latent distraction.

\section{Primary task performance}

If the operator drives inadequately when performing a secondary task, distraction is manifest. This form of distraction can be measured by using the above mentioned conflict measures. This does, however, not apply for latent distraction since readiness to respond does not necessarily translate to conflict in a low demand driving environment.

There is a way to unveil latent distraction: when a driver in an experimental setting is led into a safety-critical situation, driving behaviour can be measured. This way it becomes clear whether the driver reacts in an adequate, safe manner. But while this is a way to detect distraction, its practical use is subject to a few conditions. The experimental environment has to be manipulated and it has to be possible to measure whether the driver's reaction to the safety-critical situation is adequate. In a naturalistic driving environment it is very difficult, or even impossible, and in some cases unethical, to meet these conditions.

\section{Secondary task performance}

Secondary task performance is strongly influenced by instructions given and by individual strategies, and therefore by the motivation of the driver to perform the task at a certain level. A driver can achieve a high or low score in a secondary task, but that does not say anything about the driving task. Embedded tasks are in this sense part of the primary driving task and can therefore not be used to determine driving performance [2].

A special case is the PDT. The PDT is unobtrusive and not part of the primary driving task. Therefore it is possible to take measurements that have no effect on driving performance, but do give information about event detection and reaction times.

Several studies have demonstrated that the reaction times of distracted drivers can increase by up to 30 percent [e.g., 34]. Since hazards often require an immediate response, this is a clear impairment of the task of safe driving. Since the PDT is constantly in the background, it is 
possible to determine the driver's state for each time frame or for each change in driving situation. Previous studies also found that distraction leads to less sensitivity for hazards in the peripheral field and a decrease in amount of time spent checking the vehicle's instruments and controls [14]. The PDT measures fail in that case, as do hit rates to a stimulus (related to event detection) and reaction times (which is in itself an important aspect of driving safely). If event detection is delayed or events are even completely missed, this poses a serious threat to safe driving.

Patten and colleagues [9] studied the effects of driving in a low-demand environment while talking on a mobile telephone. Both conversation type (simple/ complex) and telephone type (handheld and hands-free) were varied, and PDT reaction times and subjective ratings of mental workload were recorded. The authors calculated the mean reaction types for each condition, and the overall percentage increase in reaction time as compared to the baseline condition. In the simple conversation condition, PDT reaction times increased on average by 12 percent. For the complex conversation, this number increased up to 45 percent. Based on this relative increase in reaction times, stopping distances were calculated, by converting the PDT reaction time (in $\mathrm{ms}$ ) and the respective velocities driven by the participants (in $\mathrm{m} / \mathrm{s}$ ) into meters used for "travelling while thinking'. These distances were added to baseline braking and stopping distances to calculate total braking and stopping distances. These distances represent the best possible scenarios, in the way that they were based on keen perception, best possible response selection, and optimal response execution [9].

Building on this method, relative decreases in drivers' readiness to respond can be calculated from PDT reaction times. In addition to the method used in [9], in which these calculations were based on a relative increase in PDT reaction times, one could also determine the seriousness of event detection impairment by taking a closer look at PDT fail and hit rates. Since PDT stimuli are given every 3 to 5 seconds throughout the whole experiment, a series of consecutive misses could indicate that the driver's focus had been narrowed for a longer period of time. This can be translated to an increased level of deterioration of the driver state.

It should be noted that, when using the PDT, instructions should be clear on the relative priorities of driving safely and the PDT, since secondary task performance is strongly influenced by the motivation of the driver [23]. Furthermore, one can only draw conclusions on relative, rather than absolute increases or decreases in relation to the personal baseline. However, as increases in reaction times can be translated to an increase in brake reaction times or stopping distances, a relative decrease in readiness to respond can be deducted from the PDT.

\section{Subjective measures}

As was described before, subjective measures can give an accurate measure of perceived mental workload. However, for the measurement and prediction of distraction effects, it is needed that either subjective measures of mental workload can be translated to performance decrements, or that subjective ratings of distraction are strongly related to actual distraction effects. Unfortunately, certain studies show otherwise. In order to determine how wellcalibrated drivers are with respect to performance decrements from distracting tasks, a testtrack study was conducted [11]. Drivers completed a series of mental arithmetic tasks on a hand-held or hands-free cell phone while driving in an instrumented vehicle. Subjective estimates of performance decrements were compared to actual performance decrements (as 
measured by lane keeping, speed control and brake response time). Drivers' performance suffered in all dual-task conditions, and their subjective ratings indeed showed a lower performance rating for dual-task conditions compared to baseline driving. However, drivers were generally not well-calibrated to the magnitude of the distraction. These errors did not stem from overconfidence in safety and skill, since the measures taken for these variables were not statistically related. In some cases, estimates of distraction were even opposite to the observed effects (i.e., smaller estimates of distraction corresponded to larger performance deficits). This was mostly the case in the highest workload conditions [11].

It can be concluded that driver distraction can not be based only on subjective measures, and that these measures, if used, should always be combined with other (reliable and sensitive) measures of distraction.

\section{Physiological measures}

Physiological measures, such as heart rate or heart rate variability, can give an indication of a change in mental workload, by showing to what extent the driver is exerting effort into the driving task. As [23] shows, different types of physiological measures are sensitive to different regions of task demands. Physiological measures are most sensitive to changes in the A3 and DB region, indicating an investment in effort and the beginning of a deterioration in performance. However, a change in physiological measures does not necessarily mean that driving performance deteriorates. Physiological measures are therefore not suitable for use as a stand-alone assessment method of driver distraction, and a combination with other assessment techniques should be made.

\section{MENTAL WORKLOAD AND DISTRACTION}

Looking at the similarity between the assessment methods of driver distraction and mental workload one would be inclined to conclude that they are highly similar. Several studies actually equate an increase in mental workload to distraction. But this is not necessarily correct and some reservations would be in order.

\section{Presence of secondary task}

Hoedemaeker and colleagues conducted a driving simulator study in which lane width and maximum speed were varied [35]. No additional task was introduced. Therefore, any effect seen could only be as a result of the primary driving task and not of any competing activity. They showed that the percentage of highly frequent steering movements, a measure of effort exerted and therefore a measure for adequate performance of the primary task, was significantly higher in the narrowest lane. Furthermore, subjective ratings (BSMI, Dutch rating scale for mental effort) confirm that driving was experienced to be more strenuous on narrow lanes and with increasing speed [35]. This is an example of a study in which mental workload was manipulated and measured, rather than distraction.

\section{Motivation and engagement}


The sole presence of the secondary task may increase task demands, but the motivation of the drivers to perform well in a certain task can have great influence on the degree to which drivers 'allow' themselves to be distracted. As De Waard [23] asserts, the instructions that are given to the driver are of great influence on the execution of the secondary task, and hence also on the performance of safe driving. Because motivation plays a role in addition to task demands and workload, it can not be stated that an increase in mental workload directly translates into driver distraction. After all, distraction, by definition, leads to decreased driving performance, and that is not necessarily the case.

As it turns out, drivers distinguish between different types of secondary tasks, even without instructions, and safe driving is a key factor in making this distinction. Cnossen and her colleagues [36] hypothesized that the nature of the secondary task may be important, and that secondary tasks serving the driving task (e.g., map reading) could receive higher priority than other secondary tasks (e.g., tuning the radio). Drivers were asked to perform a working memory task (irrelevant for driving) and a map reading task (relevant for driving). In high demand conditions, the irrelevant task was indeed neglected, but map reading resulted in more swerving, indicating that the subjects looked at the map despite the high task demands. Apparently, drivers protect high-priority task goals (safe driving) at the cost of lower-priority, distracting tasks.

A recent meta-analysis of effects of mobile telephone use on driving performance [37] showed that conversation tasks showed greater costs in driving performance than did information processing tasks. This may be due to the greater "engagement" associated with actual conversations [37]. Furthermore, McKnight and McKnight [38] compared concurrent driving and conversing at two complexity levels. They found that intense conversations were significantly more distracting than casual conversations. The level of distraction was measured by the proportion of appropriate responses to situations that required a speed or direction alteration, such as decelerating lead vehicle. The authors suggest that this significant difference between the two types of conversations is due to stronger engagement of the drivers in this complex conversation task [38].

It can be concluded that a driver's motivation to perform well in a certain task and the related engagement in a secondary task influences whether or not the driver 'allows' him- or herself to be distracted. The primary task of safe driving can be protected by the driver, and this does not necessarily have to be connected to a certain level of mental workload.

It should therefore be noted that mental workload and distraction are not the same, and that manipulating mental workload does not necessarily lead to driver distraction. Therefore it is of the utmost importance that the distinction between the two concepts is upheld, both in research question and experimental designs. To this end, it would be very useful to establish uniformity in definitions of driver distraction, as stated before. This should be a focal point in further research.

\section{CONCLUSIONS}

Mental workload and distraction, although linked when drivers perform secondary tasks while driving, are two different concepts and should be treated as different phenomena in research questions and experimental design. Increasing workload in itself is not an indication of distraction. Mental workload can increase without affecting distraction, for example when a 
driver is motivated to execute the primary driving task correctly and safely. The level of engagement in a secondary task also plays a key role in the effects on the primary driving task. Finally, the fact that workload can increase by an increasing complexity of the driving environment or as a result of changing driver state, also shows that mental workload is distinct from distraction.

The assessment of distraction should be done using a combination of different measurement techniques. Adequate or safe driving, a direct indication of distraction when induced by the presence of a secondary task, can be determined by conflict measures. These measures represent the number and seriousness of conflicts drivers encounter due to their driving style. However, although distraction can be manifested in unsafe driving behaviour, unsafe driving is not the only measure on the basis of which it can be concluded that a driver is distracted. As driving is full of possible hazards, readiness to respond is also an important aspect of safe driving. Because it is difficult to measure (latent) distraction when a driver is driving safely while executing a secondary task or in the presence of a possible distractor, using a combination of multiple measures is strongly advised. Certain measures of mental workload can be used as an indication of the level of performance in a situation that is more complex and unexpected. The Peripheral Detection Task (PDT) is a promising measure for readiness to respond and event detection, provided that it is being interpreted correctly. Further research should focus on studying these possibilities in more depth, as well as on reaching uniformity in both definition and operationalization of driver distraction.

\section{ACKNOWLEDGEMENTS}

This study has been conducted within the research centre Applications of Integrated Driver Assistance (AIDA) at the Centre for Transport Studies (University of Twente) and TNO the Netherlands, and as part of the Intelligent Vehicles project of the TRANSUMO program. TRANSUMO (TRANsition toward SUstainable MObility) is a Dutch platform for companies, governments and knowledge institutes that cooperate in the development of knowledge with regard to sustainable mobility.

\section{REFERENCES}

[1] Wang, J.-S., R.R. Knipling, M.J. Goodman (1996). The role of driver inattention in crashes: New statistics from the 1995 Crashworthiness Data System. In 40th Annual Proceedings of the Association for the Advancement of Automotive Medicine. Vancouver, British Columbia. pp. 377-392.

[2] Brookhuis, K.A., G. De Vries, D. De Waard (1991). The effects of mobile phone telephoning on driving performance, Accident Analysis and Prevention, vol. 32, pp. 309-316.

[3] Redelmeier, D.A, R.J. Tibshirani (1997). Association between cellular-telephone calls and motor vehicle crashes, The New England Journal of Medicine, vol. 336, no. 7, pp. 453-458.

[4] McEvoy, S.O., M.R. Stevenson, A.T. McCartt, M. Woodward, C. Haworth, P. Palamara, R. Cercarelli (2005). Role of mobile phones in motor vehicle crashes resulting in hospital attendance; A case-crossover study, British Medical Journal, vol. 331.

[5] Dragutinov, N., D. Twisk (2005). Use of mobile phones while driving - effects on road safety. A literature review, Dutch Institute for Road Safety Research SWOV, Report no. R2005-12, Leidschendam, the Netherlands. 
[6] Eby, D.W., J.M. Vivoda, (2003). Driver hand-held mobile phone use and safety belt use, Accident Analysis and Prevention, vol. 35, no. 6, pp. 893-895.

[7] Lamble, D., T. Kauranen, M. Laakso, H. Summala (1999). Cognitive load and detection thresholds in car following situations: Safety implications for using mobile (cellular) telephones while driving, Accident Analysis and Prevention, vol. 31, 617-623.

[8] Hancock, P.A., M.F Lesch, L. Simmons (2003). The distraction effects of phone use during a crucial driving maneuver, Accident Analysis and Prevention, vol. 35, pp. 501-514.

[9] Patten, C.J.D., A. Kircher, J. Ostlund, L. Nilsson (2004). Using mobile telephones: Cognitive workload and attention resource allocation, Accident Analysis and Prevention, vol. 36, pp. 341-350.

[10] Anttila, V., J. Luoma (2005). Surrogate in-vehicle information systems and driver behaviour in an urban environment: A field study on the effects of visual and cognitive load, Transportation Research Part F, vol. 8, pp 121-133.

[11] Horrey, W.J., M.F. Lesch, A. Garabet (2008). Assessing the awareness of performance decrements in distracted drivers, Accident Analysis and Prevention, vol. 40, no. 2, pp. 675682.

[12] Horrey, W.J., M.F. Lesch (2009). Driver-initiated distractions: Examining strategic adaptation for in-vehicle task initiation, Accident Analysis and Prevention, vol. 41, pp. 115122.

[13] Young, K., M. Regan (2007). Driver distraction: A review of the literature. in I.J. Faulks, M. Regan, M. Stevenson, J. Brown, A. Porter and J.D. Irwin (eds.), Distracted driving, Sydney, NSW: Australasian College of Road Safety.

[14] Harbluk, J.L., Y.I. Noy, M. Eizenman (2002). The impact of cognitive distraction on driver visual behaviour and vehicle control (TP No. 13889 E). Ottowa, Canada: Transport Canada.

[15] Chisholm, S.L., J.K. Caird, J. Lockhart (2008). The effects of practice with MP3 players on driving performance, Accident Analysis and Prevention, vol. 40, pp. 704-713.

[16] Treat, J.R. (1980). A study of pre-crash factors involved in traffic accidents, HSRI Research Review, vol. 10, no. 11, pp. 1-35.

[17] Sheridan, T.B. (2004). Driver distraction from a control theory perspective, Human Factors, vol. 46, no. 4, pp. 587-599.

[18] Lee, J., K. Young, M. Regan (2008). Defining driver distraction, in M. Regan, J. Lee, and K. Young (eds), Driver Distraction. Theory, Effects, and Mitigation, London: Taylor \& Francis.

[19] Young, K., M. Regan, M. Hammer (2003). Driver distraction: a review of the literature. Report no. 206, Monash University Accident Research Centre, Australia.

[20] Amundsen, F., C. Hydén (1977). Proceedings of First Workshop on Traffic Conflicts, Oslo, Norway, Institute of Transport Economics.

[21] Van der Horst, A.R.A. (1990). A time-based analysis of road user behaviour in normal and critical encounters. Doctoral Thesis, TU Delft, Delft, the Netherlands.

[22] Chin, H.-C., S.-T. Quek (1997). Measurement of traffic conflicts. Safety Science, vol. 26, no. 3, pp. 169-185. 
[23] De Waard, D. (1996). The measurement of drivers' mental workload, PhD thesis, University of Groningen. Haren, The Netherlands: University of Groningen, Traffic Research Centre.

[24] Alm, H., L. Nilsson (1994). Changes in driving behaviour as a function of handsfree mobile phone - a simulator study, Accident Analysis and Prevention, vol. 26, no. 4, pp. 441451.

[25] Lansdown, T.C., N. Brook-Carter, T. Kersloot (2004). Distraction from multiple invehicle secondary tasks: vehicle performance and mental workload implications, Ergonomics, vol. 47, no. 1, pp. 91-104.

[26] Horrey, W.J., D.J. Simons (2007). Examining cognitive interference and adaptive safety behaviours in tactical vehicle control, Ergonomics, vol. 50, no. 8, pp. 1340-1350.

[27] Van Winsum, W., M.H. Martens, L. Herland (1999). The effects of speech versus tactile driver support messages on workload, driver behaviour and user acceptance. Report no. TM99-C043). Soesterberg, Netherlands: TNO Human Factors.

[28] Van der Horst, A.R.A. \& M.H. Martens (2009). The Peripheral Detection Task (PDT): Online Measurement of Driver Cognitive Workload and Selective Attention.. SAE Special Issue 2008 Driver Metrics Workshop. (in press).

[29] Jahn, G., A. Oehme, J. Krems, C. Gelau (2005). Peripheral detection as a workload measure in driving: Effects of traffic complexity and route guidance system use in a driving study, Transportation Research Part F, vol. 8, pp. 255 - 275.

[30] Hart, S.G., L. E. Staveland (1988). Development of NASA-TLX (Task Load Index): Results of empirical and theoretical research, in P.A. Hancock and N. Meshkati (eds.) Human Mental Workload, Amsterdam: North Holland Press, pp. 239-250.

[31] Zijlstra, F.R.H. (1993). Efficiency in work behavior. A design approach for modern tools, PhD thesis, Delft University of Technology. Delft, The Netherlands: Delft University Press.

[32] Van Winsum, W., M. Hoedemaeker (2000). A road test of a prototype satellite system for in-vehicle menu control, Report no. TM-00-C003, TNO Human Factors Research Institute, Soesterberg, The Netherlands.

[33] Eggemeier, F.T., G.F. Wilson (1991). Performance-based and subjective assessment of workload in multi-task environments, in D.L. Damos (ed.), Multiple-task performance, London: Taylor \& Francis.

[34] Brookhuis, K.A., Waard, D. de, Mulder, L.J.M. (1994). Measuring driving performance by car-following in traffic. Ergonomics, vol. 37, pp. 427-434.

[35] Hoedemaeker, M., W.H. Janssen, R.F.T. Brouwer (2002). Evaluatie versmalde dwarsprofielen op het hoofdwegennet. Deelrapport 3: De relatie tussen de inrichtingsvorm en het rijgedrag, Report TM-02-C021, TNO Human Factors Research Institute, Soesterberg, The Netherlands.

[36] Cnossen, F., T. Meijman, T. Rothengatter (2004). Adaptive strategy changes as a function of task demands: a study of car drivers, Ergonomics, vol. 47, no. 2, pp. 218-236.

[37] Horrey, W.J., C.D. Wickens (2006). Examining the impact of cell phone conversations on driving using meta-analytic techniques, Human Factors, vol. 48, no. 1, pp. 196-205.

[38] McKnight, A.J., A.S. McKnight (1993). The effect of cellular phone use upon driver attention, Accident Analysis and Prevention, vol. 25, no. 3, pp. 259-265. 